\title{
On the curvature of guided surfaces
}

\author{
K. Karčiauskas and J. Peters \\ Department of Mathematics and Informatics, Vilnius University \\ Dept C.I.S.E., University of Florida
}

\begin{abstract}
Following [KP07], we analyze surfaces arising as an infinite sequence of guided $C^{2}$ surface rings. However, here we focus on constructions of too low a degree to be curvature continuous also at the extraordinary point. To characterize shape and smoothness of such surfaces, we track a sequence of quadratic functions anchored in a fixed coordinate system. These 'anchored osculating quadratics' are easily computed in terms of determinants of surface derivatives. Convergence of the sequence of quadratics certifies curvature continuity. Otherwise, the range of the curvatures of the limit quadratics gives a measure of deviation from curvature continuity.
\end{abstract}

Key words: curvature continuity, anchored osculating quadratic, guided surface,

\section{Introduction and Motivation}

The paper [KP07] introduces a framework for the construction of $C^{2}$ surfaces from surface rings $\mathbf{x}^{m}, m=0,1, \ldots$ as illustrated in Figure 1. To set the stage, we quickly review the key concepts and notations. Each surface ring $\mathrm{x}^{m}$ joins smoothly and without overlap with its predecessor $\mathbf{x}^{m-1}$ if $m>0$ and with existing boundary data if $m=0$. The sequence of rings contracts towards a central point $\mathrm{x}^{\infty}$. The ring structure is inherited from a ct-

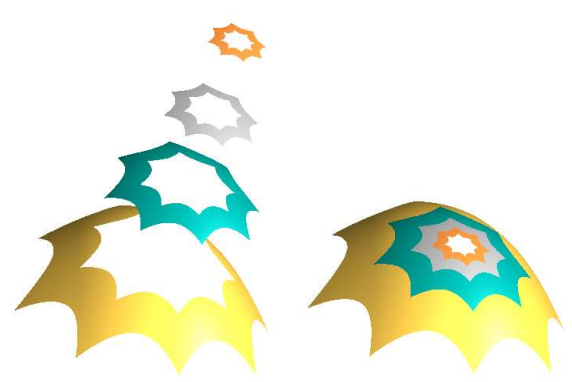

Fig. 1. Sequence of guided rings $\mathrm{x}^{m}$ and guided surface [KP07]. map (concentric tesselation map) $\rho: \Sigma \times$

Email address: jorg@cise.ufl.edu, kestutis.karciauskas@mif .vu. It (K. Karčiauskas and J. Peters).

URL: http: //www.cise.ufl. edu/ jorg (K. Karčiauskas and J. Peters). 
$\{1, \ldots, n\} \rightarrow \mathbb{R}^{2}$. That is, a ct-map embeds $n$ copies of a domain piece $\Sigma$ into the plane. $\Sigma$ is for example a square or a square with a cutout. The scaled copies of $\rho$, $\lambda^{m-1} \rho$ and $\lambda^{m} \rho$ for $0<\lambda<1$, join with prescribed continuity and tesselate the neighborhood of the origin by a sequence of annuli. Table 1 lists several ct-maps defined in [KP07] and their combinations with operators $H$ explained below. The shape of a guided surface is inherited from a guide map $\mathrm{g}: \mathbb{R}^{2} \rightarrow \mathbb{R}^{3}$ by 'sampling' the composition of $\mathbf{g}$ with the scaled copies of the ct-map. The guide $\mathrm{g}$ is a surface piece that outlines the intended shape, but need not fit the requirements of a modeling pipeline or match smoothly with the existing data. Specifically,

$$
\mathbf{x}^{m}:=H\left(\mathbf{g} \circ \lambda^{m} \rho\right)
$$

Here $H$ samples jets, i.e. a collection of derivatives, at fixed parameter values of its argument, and constructs $\mathbf{x}^{m}$ so that the resulting rings join smoothly and are internally smooth.

Table 1

\begin{tabular}{|c|c|c|c|c|c|}
\hline $\begin{array}{l}\text { ct- } \\
\text { map }\end{array}$ & $\begin{array}{l}\text { degree } \\
\rho\end{array}$ & layout & ring & $\begin{array}{l}\text { degree } \\
\mathbf{x}\end{array}$ & $\begin{array}{l}\text { repro- } \\
\text { duces }\end{array}$ \\
\hline \multirow[t]{3}{*}{$\rho_{C}$} & $(3,3)$ & $\square$-sprocket & $\mathbf{x}_{C 6}$ & $(6,6)$ & 2 \\
\hline & & (eg Catmull-Clark) & $\mathbf{x}_{C 5}$ & $(5,5)$ & 1 \\
\hline & & & $\mathbf{x}_{C 3}$ & $(3,3)$ & 1 \\
\hline \multirow[t]{2}{*}{$\rho_{L}$} & 4 & $\Delta$-sprocket & $\mathbf{x}_{L 8}$ & 8 & 2 \\
\hline & & (eg Loop) & $\mathbf{x}_{L 4}$ & 4 & 1 \\
\hline \multirow[t]{3}{*}{$\rho_{p}$} & $(3,1)$ & polar & $\mathbf{x}_{p 6}$ & $(6,5)$ & 2 \\
\hline & & & $\mathbf{x}_{p 63}$ & $(6,3)$ & 2 \\
\hline & & & $\mathbf{x}_{p 3}$ & $(3,3)$ & 1 \\
\hline$\rho_{g}$ & $(2,1)$ & polar & $\mathbf{x}_{g 4}$ & $(4,3)$ & 2 \\
\hline
\end{tabular}

Some combinations of ct-maps $\rho$ and guided surface rings $\mathbf{x}$. See Figure 2 for an explanation of the entry 'layout'. The first subscript of $\mathbf{x}$ indicates the ct-map $\rho$, the second the degree of the patches generated by $H$. The ct-map $\rho_{g}$ results in $G^{2}$ surface rings, all other ct-maps in $C^{2}$ rings. The numbers $k \in\{1,2\}$ in the last column indicate that $\mathbf{x}^{m}$ reproduces $\mathbf{g}_{k} \circ \rho$ where $\mathbf{g}_{k}$ is the $k$ th order expansion of $\mathbf{g}$ at the central point.

The point of guided constructions is to decouple the definition of the shape from the final, more restrictive representation. The shape is outlined by the guide and the final representation of $\mathbf{x}^{m}$ is in terms of low-degree Bézier or B-splines. Joining an infinite sequence of spline rings yields a surface $\mathrm{x}$ with subdivision structure. Only the use of a guide surface in place of a guide (subdivision) matrix sets guided subdivision apart from the usual procedure of refining meshes (see Section 2). Theorem 1 of [KP07] showed that the limit surfaces $\mathbf{x}$ of this process are $C^{2}$, provided 
(1) the guide surface $g$ is twice continuously differentiable at $(0,0)$ and (2) the operator $H$ generates rings $\mathbf{x}^{m}$ of sufficient degree to reproduce $\mathbf{g}_{2} \circ \rho$, where $\mathbf{g}_{2}$ is the piecewise quadratic expansion of $g$ at the central point. This theorem applies to the guided surfaces with entry ' 2 ' in the last column of Table 1.
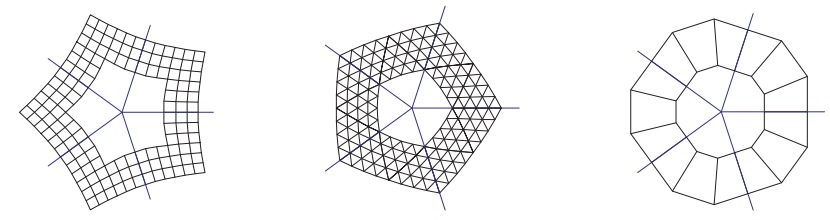

Fig. 2. Three types of ct-maps: $\rho_{C}$ ( $\square$-sprocket), $\rho_{L}$ ( $\Delta$-sprocket), $\rho_{p}$ (polar). The rays emanating from the center demarcate the segment boundaries of $\rho$ and are mapped to the segment boundaries of $g$.

The present paper focuses on the new constructions with entry ' 1 ' in the last column of Table 1. These constructions are analogous to the $C^{2}$ constructions in [KP07] and consist of a sequence of $C^{2}$ rings joining to form a $C^{2}$ surface with an ever smaller central hole. However, their degree is lower than is needed to reproduce the composition $\mathrm{g}_{2} \circ \rho$. The surfaces are therefore $C^{2}$ except in the limit extraordinary point, $\mathbf{x}^{\infty}$. We will see that the surfaces have bounded curvature and are typically visually indistinguishable from their curvature continuous siblings.

To quantify a surface's deviation from curvature continuity, we turn to a classical notion of curvature, the convergence of a sequence of quadratic functions. These quadratics are defined in a fixed coordinate system, corresponding to the tangent plane and normal at $\mathrm{x}^{\infty}$, rather than in a local coordinate system. They are therefore called 'anchored' quadratics.

Section 2 contrasts standard subdivision with guided subdivision. Section 3 introduces the new constructions, for $\square$-sprocket, $\Delta$-sprocket and polar layout, and establishes $C^{1}$ continuity of the limit surfaces. Section 4 defines curvature continuity via anchored osculating quadratics and provides curvature estimates and bounds for guided surfaces. Section 5 presents and discusses results of implementations of all three layouts.

\section{Subdivision and guides}

Subdivision has both a discrete structure, the refinement of a control net, and a continuous structure, by associating refinable generating splines with the control nodes.

The early literature on generalized subdivision (now abbreviated to subdivision) [DS78,CC78,BS86], focused exclusively on the discrete structure, namely the refinement $A^{m} \mathbf{c}$ of the control net $\mathbf{c}$ guided by a subdivision matrix $A$ (Figure 3). 

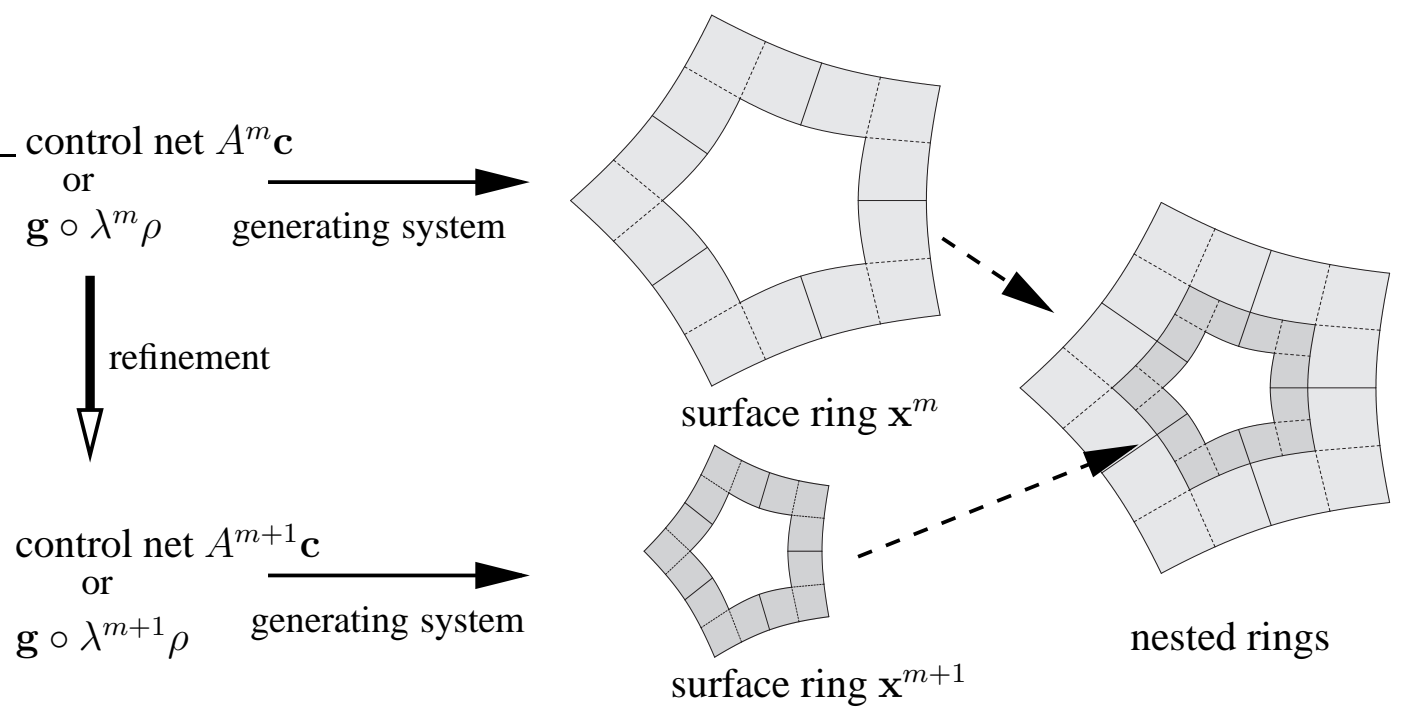

Fig. 3. Generalized subdivision and guided subdivision both generate a sequence of nested surface rings: generalized subdivision surface rings are guided by a subdivision matrix $A$, guided subdivision surface rings are guided by an explicit map g.

Alternatively, one can emphasize the generating system associated with the mesh, i.e. the spline rings that form the surface. In the early literature, these spline rings weres tacitly understood to be structurally simple spline functions so that the 'tuning' or improvement of properties of schemes focused on adjusting the spectrum of the subdivision matrix. To obtain $C^{2}$ continuity guided by a matrix, [PU98] induces zero curvature at extraordinary points; [XYD06] associate multiple values and functions with each node, achieving $C^{2}$ continuity, but only for valence three; [LL03,SW05] focus on reproduction of quadratics to combine subdivision schemes $C^{2}$ except at extraordinary points and [ZLLT06] adjusts subdivision functions to yield one $C^{2}$ extraordinary point by applying special rules for transitions across edges of the control net. Of the many $C^{1}$ curvature-bounded subdivision schemes, we implemented the constructions of [Loo02] and [ADS06] for comparison (see Section 5).

The focus of guided subdivision is the continuous structure of the generating system. This is grounded in the analytic point of view [Rei95,RP05] that characterizes subdivision as a process of piecing a surface together as nested spline rings converging to an extraordinary point (see Figure 3, right). In guided subdivision, this sequence is locally guided by a map $\mathrm{g} \circ \lambda^{m} \rho$. If the guide surface were a single, global polynomial and we dropped the operator $H$ then guided surfacing would reduce to the approach in [Pra97,Rei98]. However, such global polynomial mappings, as well as low degree piecewise polynomial mappings, are known to create surfaces with curvature flaws (see e.g. Figure 3 of [KP07]). By sampling, guided subdivision decouples the need for a low degree standardized representation from the degree of the guide. Most recently, a scheme was derived that combines the features of generalized subdivision and guided subdivision for polar layout [KMP06]. 


\section{Guided surfaces}

This section reviews the construction of guided rings $\mathrm{x}^{m}$ in [KP07]. At first sight, guided surfacing appears to be more complex than subdivision based on the control mesh. However, Observation 1 of [KP07] shows that guided surfacing based on piecewise polynomial guides is numerically stable and stationary.

Table 1 lists ct-maps and the degree of rings obtained by applying operators $H$. The operators corresponding to a ' 2 ' in the last column have been explained in detail in [KP07].

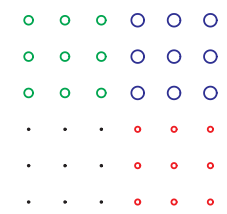

Fig. 4. $h^{5,5}$ combines four $3 \times 3$ blocks of Bézier coefficients, representing partial derivatives up to second order at the four corners, into one patch of degree $(5,5)$.

The operator $h^{5,5}$ samples (2,2)-jets at each corner (Figure 4) and combines $3 n$ such sampled patches into a ring $\mathbf{x}_{C 5}$ of degree $(5,5) . h^{5,5}$ can be replaced by an operator $h^{3,3}$ that substitutes, for each $(5,5)$ patch, a $3 \times 3$ array of bicubic patches. The formulas determining the Bézier coefficients are illustrated in Figure 5. Alternatively, we could view the $3 \times 3$ of patches as one bicubic surface piece in B-spline representation, with single knots in the interior and four-fold knots at the boundary.

Replacing the three layers of Bézier coefficients of $\mathbf{x}^{m}$ abutting $\mathbf{x}^{m-1}$ by extrapolating $\mathbf{x}^{m-1}$ ensures $C^{2}$ continuity; that is, the surface rings join $C^{2}$ when $\mathbf{x}^{m-1}$ is represented in once-subdivided form to match the granularity. The resulting type of surface ring is $\mathbf{x}_{C 3}$. Similarly, replacing the degree $(6,3)$ patches of $\mathbf{x}_{p 6}$ by three bicubics yields $\mathbf{x}_{p 3}$. A bicubic macro-patch construction corresponding to $\rho_{g}$ has also been developed, but presents no additional insight (and a second curvature-

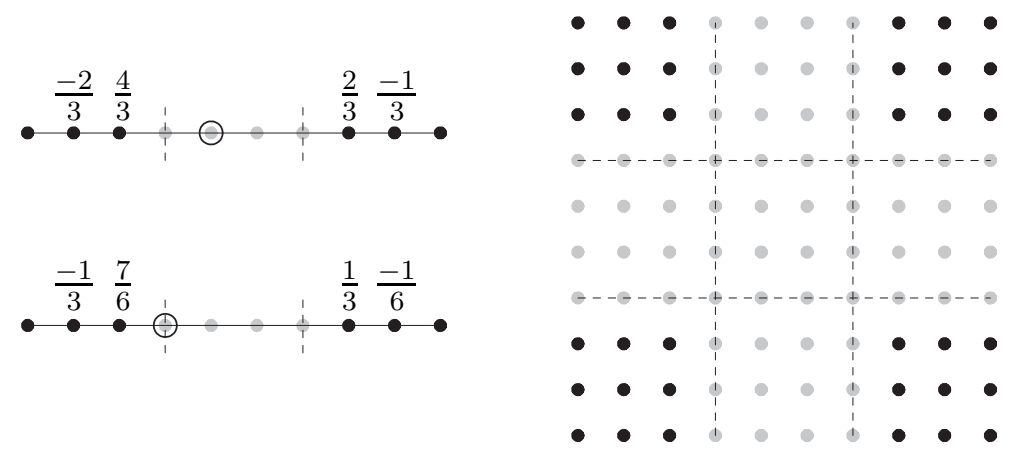

Fig. 5. Interpolation of a (2,2)-jet at the corners. (left) Stencil for Bézier coefficients to form a $C^{2}$ composite curve. (right) $\mathbf{x}_{C 3}$ construction: the $3 \times 3$ jets in Bézier form, shown as $\bullet$, are either obtained by sampling or, when adjacent to the previous ring, by subdividing the previous ring and extending it in a $C^{2}$ fashion. 
bounded construction of degree $(3,3)$ does not seem worth reporting on when the $G^{2}$ construction $\mathbf{x}_{g 4}$ uses patches of only degree $(4,3)$.)

\section{1 $C^{2}$ guided rings of total degree 4}

The construction of $\mathbf{x}_{L 4}$ is conceptually alike the previous constructions, but merits an extra section due to the different, $\Delta$-sprocket layout. The ring is defined in terms of the box-spline with directions $\left[\begin{array}{llllll}1 & 0 & 1 & 1 & 0 & 1 \\ 0 & 1 & 1 & 0 & 1 & 1\end{array}\right]$ that also underlies Loop subdivision. This box-spline is $C^{2}$ and of degree 4 . We will determine all box-spline coefficients of one ring from sampled derivatives. By construction from box-spline coefficients, the rings are $C^{2}$ and join $C^{2}$ in the sense of Loop subdivision.

The sampling operator $h^{4}$ collects eight derivatives at a sample point:

$$
\left(\partial_{s}^{j} \partial_{t}^{k} f\right)_{0 \leq j+k \leq 2}, \partial_{s}^{2} \partial_{t} f, \partial_{s} \partial_{t}^{2} f \quad f:=\mathbf{g}_{i} \circ \lambda^{m} \rho_{i} .
$$

These eight derivatives define eight box-spline coefficients (Figure 6, middle,right) and we select seven of these associated with the sample point (see Figure 7, (b)).
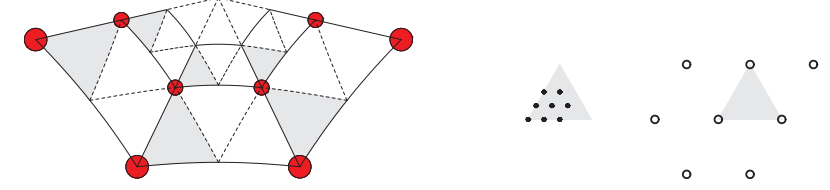

Fig. 6. (left) Locations of $h^{4}$-sampling in consecutive steps $m-1$ and $m$. (middle) representation in Bézier form and (right) representation in terms of box-spline coefficients.

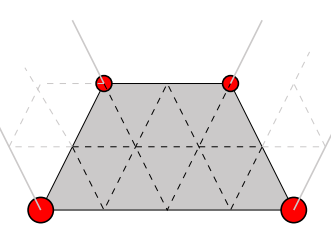

(a)

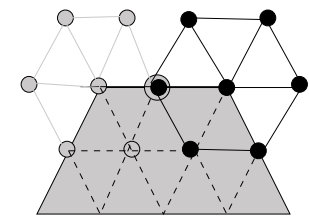

(b)

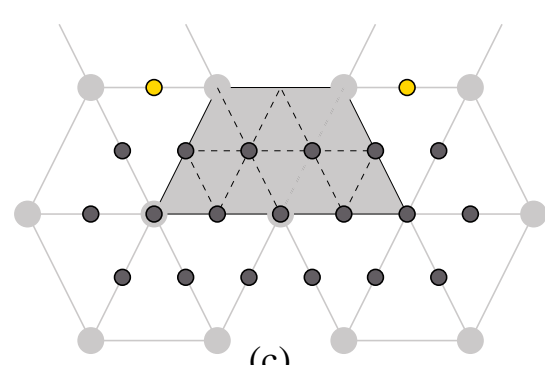

(c)

Fig. 7. Construction of one segment of a ring in grey. (a) The four sample points of a segment. Two of the sample points are associated with the previous, coarser layer $m-1$ and depicted as larger circles. (b) Seven of the eight box-spline coefficients at level $m$, determined by $h^{4}$-sampling at the upper right sample point, are depicted as $\bullet$. (c) Subdivision of the box-spline net at level $m-1$, depicted as light grey disks, yields another set of coefficients at level $m$. The two topmost coefficients are borrowed from neighboring segments.

The construction of the box-spline coefficients of one guided ring is sketched in Figure 7. At each iteration, $h^{4}$ is applied $n$ times to generate new box spline coefficients. Where these new box-spline coefficients overlap (see Figure 7 (b)), they are 
averaged. Subdivision of the box-spline net at the previous level (in step 0, of the input data) generates another set of box-spline coefficients at the same granularity as shown in Figure 7 (c). Where these coefficients overlap the newly created ones, they prevail and replace the new ones. Together (see Figure 8, left), the box-spline coefficients define a ring with $12 n$ polynomial pieces arranged as a double layer (Figure 8, right).
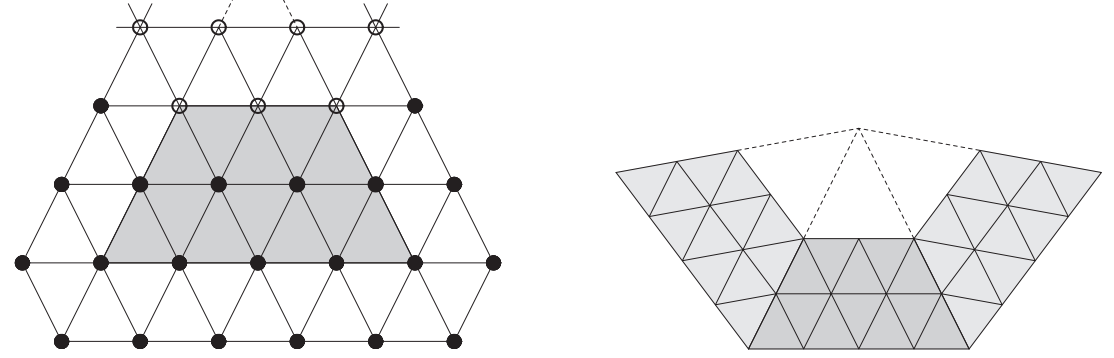

Fig. 8. Assembly of the double ring. (left) The box-spline coefficients from levels $m-1$ and $m$ determining one of $n$ segments. Coefficients marked as hollow circles $\circ$ stem from sampling at level $m$ (cf. Figure 7, left,middle). The remaining coefficients, marked as $\bullet$ were generated in level $m-1$. (right) Partial surface ring $\mathbf{x}_{L 4}$.

\section{$3.2 C^{1}$ continuity}

To analyze the surfaces in the limit, we assume that $\mathrm{g}$ is $C^{2}$ at the center point, and choose the coordinate system so that

$$
\mathbf{g}(0,0)=(0,0,0)
$$

We denote by $\mathbf{g}_{k}^{h}, k=0, \ldots, d$, the homogeneous part of degree $k$ of the guide surface cap $\mathbf{g}$. That is $\mathbf{g}_{k}^{h}$ is a linear combination of monomials of total degree $k$ in the expansion of $\mathbf{g}$ with respect to the parameters $(u, v)$ and $\mathbf{g}_{k}^{h}(\lambda \cdot)=\lambda^{k} \mathbf{g}_{k}^{h}$. Then

$$
\text { for } m>0, \quad \mathbf{x}_{k}^{m}:=H\left(\mathbf{g}_{k}^{h} \circ \lambda^{m} \rho\right)=\lambda^{m k} H\left(\mathbf{g}_{k}^{h} \circ \rho\right) \text {. }
$$

(Note that $\mathbf{x}_{k}^{m}$ is typically not homogeneous.) This yields the decomposition

$$
\mathbf{x}^{m}=\sum_{k=0}^{d} \lambda^{(m-1) k} \mathbf{x}_{k}^{1}=: \sum_{k=0}^{d} l^{k}\left(x_{k}^{h}, y_{k}^{h}, z_{k}^{h}\right) .
$$

Retracing the proof of curvature continuity in Theorem 1 of [KP07], but replacing reproduction of $\mathbf{g}_{2} \circ \lambda^{m} \rho$, where $\mathbf{g}_{2}:=\mathbf{g}_{0}^{h}+\mathbf{g}_{1}^{h}+\mathbf{g}_{2}^{h}$ by reproduction only of $\left(\mathbf{g}_{0}^{h}+\mathbf{g}_{1}^{h}\right) \circ \lambda^{m} \rho$, we see that $\mathbf{x}^{\infty}=\mathbf{g}(0,0)$, a unique limit of the tangent planes of the surface rings $\mathbf{x}^{m}$ exists as $m \rightarrow \infty$ and equals the tangent plane of the guide surface at $\mathbf{x}^{\infty}$, and the guided subdivision surface $\mathbf{x}$ is $C^{1}$ at $\mathbf{x}^{\infty}$. 


\section{Curvature Continuity and Shape Characterization}

We now use a classical parametrization-independent notion of curvature continuity. The idea is to remove the influence of the surface parameterization by fixing the local coordinate system at $(0,0)$ and isolate a quadratic map so that surface rings are a higher-order perturbation of this quadratic. This approach can also measure deviation from curvature continuity. To make the idea precise, we denote piecewise polynomial terms of total degree greater than $k$ in the variables $s_{1}, \ldots, s_{i}$ and with the coefficients depending on $t_{1}, \ldots, t_{j}$ by

$$
\pi_{>k}\left(s_{1}, \ldots, s_{i} ; t_{1}, \ldots, t_{j}\right) .
$$

If the coefficients are constant, we write $\pi_{>k}\left(s_{1}, \ldots, s_{i}\right)$.
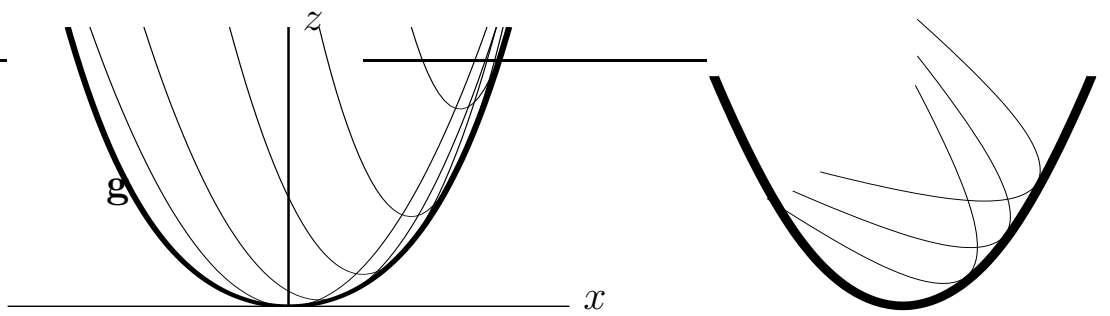

Fig. 9. Illustration in one variable: (left) anchored osculating quadratics (thin stroke parabolas) are defined with respect to a fixed coordinate system defined by position and normal of $\mathrm{g}$ (thick curve) at the central point. (right) Quadratics in local coordinates.

Definition 1 (curvature continuity) Let $\mathbf{f}: \mathbb{R}^{2} \rightarrow \mathbb{R}^{3}$ be tangent plane continuous at $\mathbf{f}_{0}:=\mathbf{f}(0,0)$. We choose the coordinate system so that $\mathbf{f}_{0}=(0,0,0)$ and the tangent plane at $\mathbf{f}_{0}$ is $\{z=0\}$. Further assume for $(s, t) \neq(0,0)$ that $(x, y, z):=$ $\mathbf{f}(s+\sigma, t+\tau)$ satisfies the equation

$$
z=\mathbf{q}(s, t) \cdot\left[x^{2}, x y, y^{2}, x, y, 1\right]^{\mathrm{t}}+\pi_{>2}(\sigma, \tau ; s, t), \quad \mathbf{q}:=\left[q_{0}, \ldots, q_{5}\right] .
$$

where $\mathbf{q}$ is a continuous function of $(s, t)$. Then

(i) $\mathbf{q}(s, t)$ is called an anchored osculating quadratic and

(ii) $\mathbf{f}$ is curvature continuous at $\mathbf{f}_{0}$ if the anchored osculating quadratics have a unique limit at $\mathbf{f}_{0}$.

Direct symbolic computation gives concrete formulas for anchored osculating quadratics. We use subscripts to indicate partial derivatives, e.g. $x_{s}:=\frac{\partial x}{\partial s}$.

Lemma 1 If $\mathbf{f}(s, t):=(x(s, t), y(s, t), z(s, t))$ then the maps $q_{j}: \mathbb{R}^{2} \rightarrow \mathbb{R}$ are

$$
\begin{aligned}
& q_{0}=\left[\begin{array}{ll}
y_{t} & y_{s}
\end{array}\right] Q\left[\begin{array}{l}
y_{t} \\
y_{s}
\end{array}\right], \quad q_{1}=-2\left[\begin{array}{ll}
x_{t} x_{s}
\end{array}\right] Q\left[\begin{array}{l}
y_{t} \\
y_{s}
\end{array}\right], \quad q_{2}=\left[\begin{array}{ll}
x_{t} x_{s}
\end{array}\right] Q\left[\begin{array}{l}
x_{t} \\
x_{s}
\end{array}\right], \\
& q_{3}=-2 q_{0} x-q_{1} y-j^{y z} / j^{x y}, \quad q_{4}=-q_{1} x-2 q_{2} y-j^{z x} / j^{x y}, \\
& q_{5}=q_{0} x^{2}+q_{1} x y+q_{2} y^{2}+\left(j^{y z} x+j^{z x} y+j^{x y} z\right) / j^{x y},
\end{aligned}
$$


where $|\cdot|$ denotes a determinant and

$$
\begin{gathered}
Q:=\frac{1}{2(j x y)^{3}}\left[\begin{array}{cc}
L & -M \\
-M & N
\end{array}\right], \quad j^{x y}:=\left|\begin{array}{ll}
x_{s} & y_{s} \\
x_{t} & y_{t}
\end{array}\right|, \\
L:=\left|\begin{array}{lll}
x_{s s} & y_{s s} & z_{s s} \\
x_{s} & y_{s} & z_{s} \\
x_{t} & y_{t} & z_{t}
\end{array}\right|, \quad M:=\left|\begin{array}{lll}
x_{s t} & y_{s t} & z_{s t} \\
x_{s} & y_{s} & z_{s} \\
x_{t} & y_{t} & z_{t}
\end{array}\right|, \quad N:=\left|\begin{array}{lll}
x_{t t} & y_{t t} & z_{t t} \\
x_{s} & y_{s} & z_{s} \\
x_{t} & y_{t} & z_{t}
\end{array}\right| .
\end{gathered}
$$

Let $(s(\tilde{s}, \tilde{t}), t(\tilde{s}, \tilde{t}))$ be a different parametrization of $(x, y, z)$. Then $\partial_{\tilde{s}} x(s, t)=$ $x_{s} s_{\tilde{s}}+x_{t} t_{\tilde{s}}$ etc.. By the multilinearity of the determinants, a lengthy but straightforward computation proves the terms $q_{j}$ to be unchanged under change of variables.

Lemma 2 The anchored osculating quadratic at a point is a geometric invariant, i.e. independent of the parametrization.

Similarly, multilinearity of determinants and elementary operations establish the following lemma where we write $q_{j}(x, y, z)$ in place of $q_{j}(x(s, t), y(s, t), z(s, t))$.

Lemma 3 If $\mathbf{f}=(x, y, z):=\left(l x_{1}+l^{2} \bar{x}, l y_{1}+l^{2} \bar{y}, l^{2} z_{2}+l^{3} \bar{z}\right)$ then

$$
\lim _{l \rightarrow 0} q_{j}(x, y, z)=q_{j}\left(x_{1}, y_{1}, z_{2}\right)
$$

For the "height function" at a point, the anchored osculating quadratic simplifies even further.

Lemma 4 If $\mathbf{f}(s, t):=(s, t, h(s, t))$ then

$$
q_{0}=\frac{1}{2} h_{s s}, \quad q_{1}=h_{s t}, \quad q_{2}=\frac{1}{2} h_{t t}
$$

The idea now is to insert $\mathbf{f}:=\mathbf{x}^{m}$ into (4) and to bound the deviation from the quadratic as $m \rightarrow \infty$.

Theorem 1 Let $\mathrm{x}$ be a guided surface of a type listed in Table 1. Then the curvature of $\mathrm{x}$ at the central point is bounded. If the entry in last column is ' 2 ' then the surface at $\mathrm{x}^{\infty}$ is curvature continuous and its anchored osculating quadratic agrees with that of the curvature continuous guide $\mathrm{g}$.

Proof We fix the coordinate system so that the tangent plane of $\mathbf{g}$ at $\mathbf{x}^{\infty}=(0,0,0)$ is $\{z=0\}$ and $\mathrm{g}$ has the expansion

$$
\mathbf{g}=\left(u+\pi_{>1}(u, v), v+\pi_{>1}(u, v), a u^{2}+b u v+c v^{2}+\pi_{>2}(u, v)\right) .
$$


In this coordinate system, applying formulas (7), (6) and (5) to $\mathbf{x}^{m}=(x, y, z)$ expanded according to (3)

$$
\mathbf{x}^{m}=\left(l x_{1}^{h}+l^{2}\left(\sum_{k=2}^{d} l^{k-2} x_{k}^{h}\right), l y_{1}^{h}+l^{2}\left(\sum_{k=2}^{d} l^{k-2} y_{k}^{h}\right), l z_{2}^{h}+l^{3}\left(\sum_{k=3}^{d} l^{k-3} z_{k}^{h}\right)\right),
$$

we obtain from Lemma 3

$$
\lim _{l \rightarrow 0} q_{j}(x, y, z)=q_{j}\left(x_{1}^{h}, y_{1}^{h}, z_{2}^{h}\right)
$$

and, since the arguments are homogenous, we can read off

$$
\lim _{l \rightarrow 0} q_{j}=0, \quad j=3,4,5
$$

Now, we define

$$
q_{j}^{\infty}:=\lim _{l \rightarrow 0} q_{j}(x, y, z)=q_{j}\left(x_{1}^{h}, y_{1}^{h}, z_{2}^{h}\right), \quad \text { for } j=0,1,2 .
$$

For type 1 schemes, i.e. schemes that do not reproduce quadratics composed with the ct-map, $q_{j}^{\infty}$ depend on $s$ and $t$ and are polynomials divided by the corresponding polynomial piece of $j^{x y}$ on each of its $k n$ domain pieces (unit triangles or squares). For example, for the scheme of line 2 of Table $1, k=3$ and for line $3, k=27$. Since $\left|j^{x y}\right|>0$ by injectivity of ct-maps and each of the $k n$ domain pieces of a $q_{j}^{\infty}$ is compact, each $q_{j}^{\infty}$ is bounded.

Since type 2 schemes reproduce quadratics,

$$
z_{2}^{h}=a\left(x_{1}^{h}\right)^{2}+b\left(x_{1}^{h} y_{1}^{h}\right)+c\left(y_{1}^{h}\right)^{2}
$$

allows us to reparameterize $q_{j}^{\infty}=q_{j}\left(s, t, a s^{2}+b s t+c t^{2}\right)$ with the coefficents of the anchored osculating quadratic of $\mathbf{g}$ at $\mathbf{x}^{\infty}$. Indeed, by Lemma $4, q_{0}^{\infty}=a, q_{0}^{\infty}=b$ and $q_{0}^{\infty}=c$. With these well defined limits at $\mathbf{x}^{\infty}$, we can apply the mean value theorem to conclude that the surface is $C^{2}$ at $\mathbf{x}^{\infty}$.

The theorem yields an alternative proof of curvature continuity of the surfaces considered in [KP07]. Moreover, it gives a concrete way to compute bounds.

\subsection{Computing Curvature Bounds}

Specifically, we compute as follows.

(i) Bound the coefficients $q_{0}, q_{1}, q_{2}$ for the functions $f_{1}:=u^{2}, f_{2}:=u v, f_{3}:=v^{2}$. This yields nine intervals that allow computing the bounds for any function $f=$ $\gamma_{1} u^{2}+\gamma_{2} u v+\gamma_{3} v^{2}$. 
(ii) If $(x, y, z)$ is an orthogonal coordinate system in $\mathbb{R}^{3}$ with $\mathbf{x}^{\infty}=(0,0,0)$, tangent plane $\{z=0\}$ at $\mathbf{x}^{\infty}$ and $\mathbf{g}=\left(e_{11} u+e_{12} v+\pi_{>1}(u, v), e_{21} u+e_{22} v+\right.$ $\left.\pi_{>1}(u, v), a u^{2}+b u v+c v^{2}+\pi_{>2}(u, v)\right)$. Then the bounds of the coefficients $q_{k}^{\prime}$ of the osculating quadratic in such system are calculated from the previous bounds on coefficients $q_{k}$ as

$$
\begin{aligned}
& q_{0}^{\prime}=q_{0} e_{0}^{2}+q_{1} e_{0} e_{1}+q_{2} e_{1}^{2}, q_{2}^{\prime}=q_{0} e_{2}^{2}+q_{1} e_{2} e_{3}+q_{2} e_{3}^{2} \\
& q_{1}^{\prime}=2 q_{0} e_{0} e_{2}+q_{1}\left(e_{0} e_{3}+e_{1} e_{2}\right)+2 q_{2} e_{1} e_{3}
\end{aligned}
$$

where $\left(\begin{array}{cc}e_{0} & e_{2} \\ e_{1} & e_{3}\end{array}\right):=\left(\begin{array}{ll}e_{11} & e_{12} \\ e_{21} & e_{22}\end{array}\right)^{-1}$

(iii) To bound Gaussian and mean curvatures, we observe that for $\left(x, y, q_{0}^{\prime} x^{2}+q_{1}^{\prime} x y+\right.$ $\left.q_{2}^{\prime} y^{2}\right)$ the mean curvature $H$ and the Gaussian curvature $K$ at the origin $(0,0,0)$ are

$$
H=q_{0}^{\prime}+q_{2}^{\prime}, K=4 q_{0}^{\prime} q_{2}^{\prime}-q_{1}^{\prime 2} .
$$

Substituting formulas (16), we get

$$
\begin{aligned}
& H=\left(e_{0}^{2}+e_{2}\right)^{2} q_{0}+\left(e_{0} e_{1}+e_{2} e_{3}\right) q_{1}+\left(e_{1}^{2}+e_{3}^{2}\right) q_{2}, \\
& K=\left(e_{0} e_{3}-e_{1} e_{2}\right)^{2}\left(4 q_{0} q_{2}-q_{1}^{2}\right) .
\end{aligned}
$$

For Gaussian curvature $K$, we get a tighter estimate if, in formula (17), the part $4 q_{0} q_{2}-q_{1}^{2}$ is precomputed with respect basis functions $f_{1}, f_{2}, f_{3}$. Consistent with [PU01], there are six precomputed intervals for $k$ and nine for $H$.

(iv) Defining $f_{1}:=u^{2}+v^{2}, f_{2}:=u v, f_{3}:=v^{2}$ gives an immediate impression of how guided subdivision reproduces canonical elliptic, hyperbolic and parabolic shapes. Table 4.1 lists bounds on the Gaussian curvature for these shapes when $n=8$.

Table 2

Gauss curvature bounds on monomials for different $\mathrm{x}$ and $n=8$.

\begin{tabular}{|l|l|ccc|}
\hline guide & $K$ & $K\left(\mathbf{x}_{C 3}\right)$ & $K\left(\mathbf{x}_{C 5}\right)$ & $K\left(\mathbf{x}_{p 3}\right)$ \\
\hline$u^{2}+v^{2}$ & 4 & {$[3.87501,4.22213]$} & {$[3.96505,4.02945]$} & {$[3.98505,4.03352]$} \\
$u v$ & -1 & {$[-1.06493,-0.96478]$} & {$[-1.00426,-0.99261]$} & {$[-1.05229,-0.97047]$} \\
$v^{2}$ & 0 & {$[-0.15738,0.15644]$} & {$[-0.02054,0.02241]$} & {$[-0.09641,0.05184]$} \\
\hline
\end{tabular}

\section{Discussion}

Traditional 'tuning' of subdivision algorithms focussed on explicitly setting eigenvalues or on formulating an optimization problem to approximate several spectral properties (e.g. [BK04]). More recently, subsubdominant eigenvectors have gained attention in the context of optimizing surfaces with respect to shape charts [KPR04,ADS06,GU06]. This allows enforcing, for example, convexity. In guided 
subdivision we prescribe, via the guide surface, both the dominant eigenvalues and the dominant eigenvectors up to the order of continuity. Moreover, prescribing the guide surface yields a regional averaging as opposed to the very localized averaging of standard subdivision rules. To set the guided constructions in perspective, Figures 11, 12 and 13 compare the Gauss curvature to two recently published tuned curvature-bounded surface constructions. Overall, the shape of guided surfaces appears to be the most predictable of the constructions.

In the 2005 version of this paper [KP05], we observed that, by increasing the sampling density at each step, we can build guided surfaces with subdivision structure that are $C^{2}$ and of degree $(3,3)$. This approach is theoretically interesting in that it sheds new light on the challenge to build $C^{2}$ subdivision surfaces of the degree of Catmull-Clark surfaces and on the lower bounds on the degree of curvature continuous subdivision surfaces [Rei96,Pra98]. The approach is however at present not practical since the patch size shrinks rapidly and the number of patches grows too fast.

We have, however been able to implement and thus practically test all low-degree guided constructions presented in this paper for all currently used subdivision layouts: $\square$-sprocket, $\Delta$-sprocket and polar. While the combination of degree and patch layout is too low to be curvature continuous at the extraordinary point, the surfaces are $C^{2}$ everywhere else and their curvature is bounded. In fact, the surfaces are typically visually indistinguishable from their curvature continuous siblings defined in [KP07]. In Figure 10 and Table 4.1 we therefore resort to Gauss-curvature images to illustrate the trade-off and effect of choosing low-degree approximations. We note that the lower the degree, the more fractured the distribution of curvature.

While high-end engineering applications will require excellent curvature distribution, we expect that the low-degree constructions will be useful for high-end rendering where a good visual impression suffices.

Acknowledgement: This work was supported by NSF Grants CCF-0430891 and DMI-0400214.

\section{References}

[ADS06] U. H. Augsdörfer, N. A. Dodgson, and M. A. Sabin. Tuning subdivision by minimising gaussian curvature variation near extraordinary vertices. Computer Graphics Forum (Proc. Eurographics), 25(3):263-272, 2006.

[BK04] Loïc Barthe and Leif Kobbelt. Subdivision scheme tuning around extraordinary vertices. In Computer Aided Geometric Design, pages 561-583, 2004.

[BS86] A.A. Ball and D.J.T. Storry. A matrix approach to the analysis of recursively generated B-spline surfaces. Computer Aided Design, 18:437-442, 1986. 


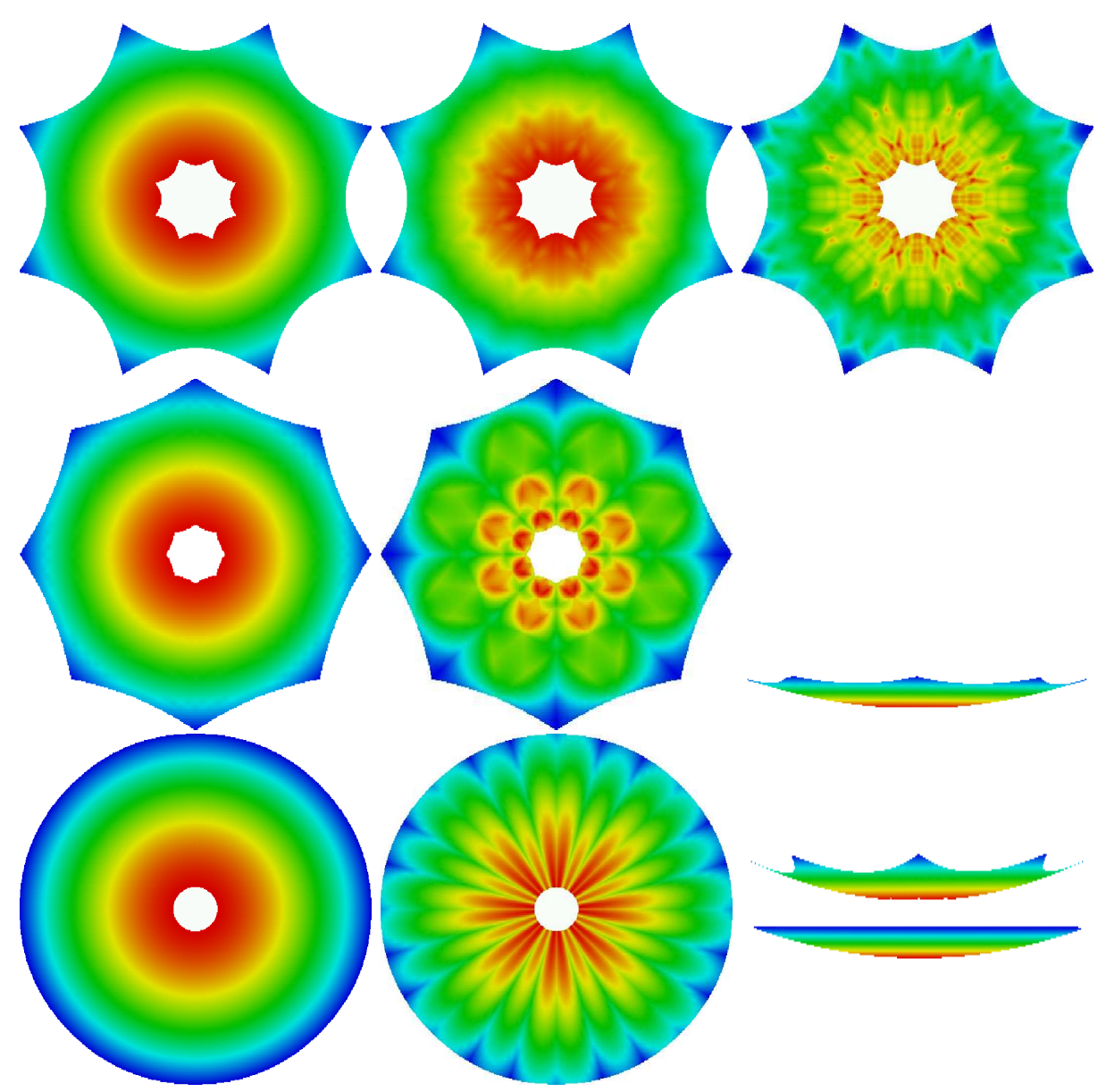

Fig. 10. Curvature-distribution- $v s$-degree trade-off. The guide for the examples is $\mathbf{g}:=\left(x, y, x^{2}+y^{2}\right)$. The left column corresponds to schemes reproducing $\mathbf{g}_{2} \circ \rho$. All figures are shaded according to Gauss curvature with the curvature values evenly spread over the red-green-blue range. (top row) $\mathbf{x}_{C 6}, \mathbf{x}_{C 5}$ and $\mathbf{x}_{C 3} ;$ (middle row) $\mathbf{x}_{L 8}, \mathbf{x}_{L 4} ;$ (bottom row) $\mathbf{x}_{g 4}, \mathbf{x}_{p 3}$. The additional side views show (middle row) $\mathbf{x}_{L 4}$ and (bottom row) $\mathbf{x}_{C 6}$ (top) and $\mathbf{x}_{g 4}$.

[CC78] E. Catmull and J. Clark. Recursively generated B-spline surfaces on arbitrary topological meshes. Computer Aided Design, 10:350-355, 1978.

[DS78] D. Doo and M. Sabin. Behaviour of recursive division surfaces near extraordinary points. Computer Aided Design, 10:356-360, September 1978.

[GU06] I. Ginkel and G. Umlauf. Loop subdivision with curvature control. In A. Scheffer and K. Polthier, editors, Proceedings of Symposium of Graphics Processing (SGP), June 26-28 2006, Cagliari, Italy, pages 163-172. ACM Press, 2006.

[KMP06] K. Karčiauskas, A. Myles, and J. Peters. A $C^{2}$ polar jet subdivision. In A. Scheffer and K. Polthier, editors, Proceedings of Symposium of Graphics Processing (SGP), June 26-28 2006, Cagliari, Italy, pages 173-180. ACM Press, 2006.

[KP07] K. Karčiauskas and J. Peters. Concentric tesselation maps and curvature continuous guided surfaces. Computer Aided Geometric Design, 24(2):99-111, 
Feb 2007.

[KP05] K. Karčiauskas and J. Peters. Guided Subdivision, manuscript, June 2005, http://www.cise.ufl.edu/research/SurfLab/papers/05guiSub.pdf

[KPR04] K. Karčiauskas, J. Peters, and U. Reif. Shape characterization of subdivision surfaces - case studies. Computer Aided Geometric Design, 21(6):601-614, july 2004 .

[LL03] Adi Levin and David Levin. Analysis of quasi-uniform subdivision. Appl Comp Harm Anal, (15):18-32, 2003.

[Loo02] C. Loop. Bounded curvature triangle mesh subdivision with the convex hull property. The Visual Computer, 18(5-6):316-325, 2002.

[Pra97] H. Prautzsch. Freeform splines. Comput. Aided Geom. Design, 14(3):201-206, 1997.

[Pra98] Hartmut Prautzsch. Smoothness of subdivision surfaces at extraordinary points. Adv. Comput. Math., 9(3-4):377-389, 1998.

[PU98] Hartmut Prautzsch and Georg Umlauf. Improved triangular subdivision schemes. In Computer Graphics International, pages 626-632, 1998.

[PU01] J. Peters and G. Umlauf. Computing curvature bounds for bounded curvature subdivision. Computer Aided Geometric Design, pages 455-462, 2001.

[Rei95] U. Reif. A unified approach to subdivision algorithms near extraordinary vertices. Comp Aided Geom Design, 12:153-174, 1995.

[Rei96] U. Reif. A degree estimate for subdivision surfaces of higher regularity. Proc. Amer. Math. Soc., 124(7):2167-2174, 1996.

[Rei98] U. Reif. TURBS-topologically unrestricted rational $B$-splines. Constr. Approx., 14(1):57-77, 1998.

[RP05] U. Reif and J. Peters. Topics in multivariate approximation and interpolation. In K. Jetter et al., editor, Structural Analysis of Subdivision Surfaces - A Summary, pages 149-190. Elsevier Science Ltd, 2005.

[SW05] Scott Schaefer and Joe D. Warren. On $\mathrm{C}^{2}$ triangle/quad subdivision. ACM Trans. Graph, 24(1):28-36, 2005.

[XYD06] Y. Xue, T. P.-Y. Yu, and T. Duchamp. Jet subdivision schemes on the $k$-regular complex. Comp Aided Geom Design, 23(4):361-396, 2006.

[ZLLT06] A. Zulti, A. Levin, D. Levin, and M. Teicher. $C^{2}$ subdivision over triangulations with one extraordinary point. Comp Aided Geom Design, 23(2):157-178, feb 2006. 

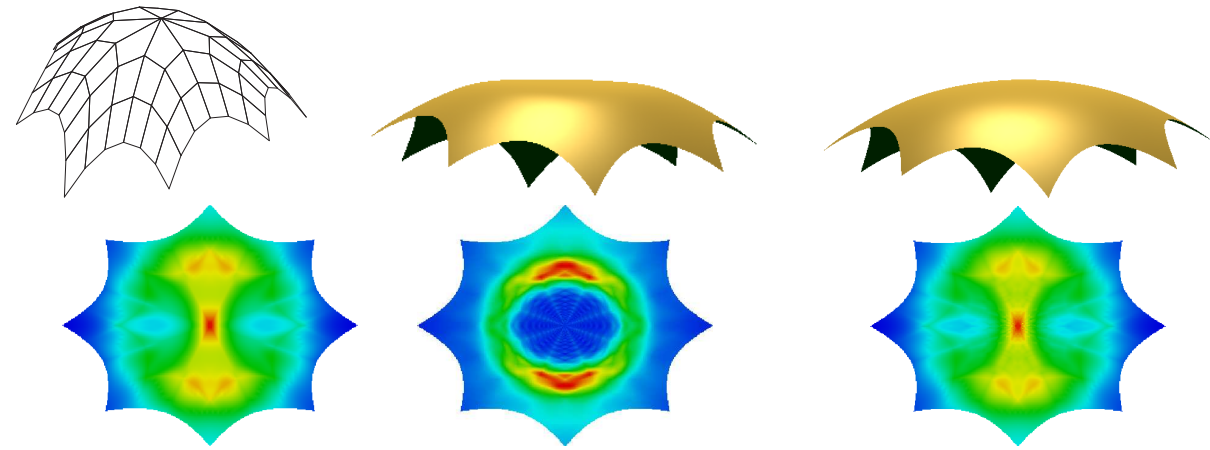

Fig. 11. Constructions of degree (3,3). (top row, left) Input mesh: the characteristic control net of Catmull-Clark subdivision projected onto the paraboloid $z=-x^{2}-0.6 y^{2}$; (middle) Tuned construction of [ADS06]; (right) guided construction $\mathbf{x}_{C 3}$. (bottom row) Gaussian curvature shading: the $C^{2}$ construction $\mathbf{x}_{C 6}$ (for comparison), [ADS06], $\mathbf{x}_{C 3}$.
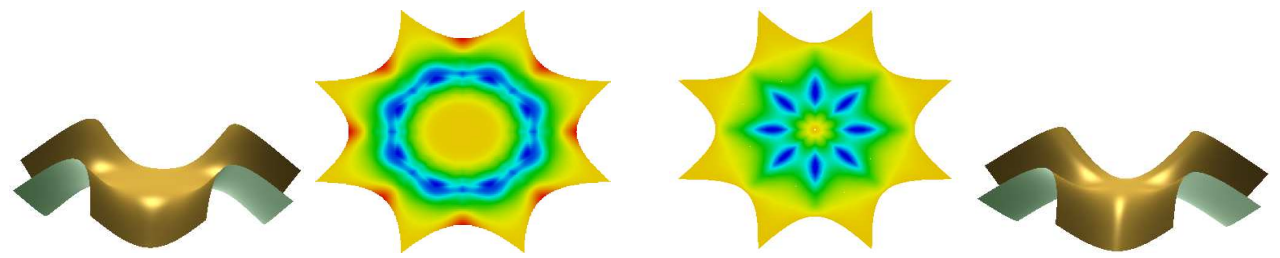

Fig. 12. Constructions of degree $(3,3)$ for double-saddle data. (left) Tuned construction of [ADS06]: Gauss curvature is both negative and positive (red, where segments meet). (right) guided construction $\mathbf{x}_{C 3}$ is non-positive as it should be.
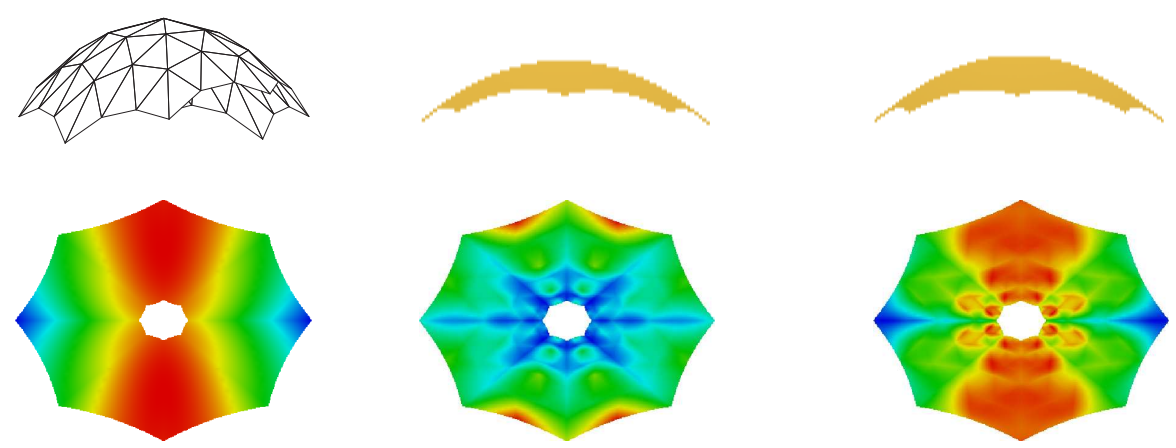

Fig. 13. Constructions of total degree 4. (top row, left) Input mesh: the characteristic control net of Loop subdivision projected onto the paraboloid $z=-x^{2}-0.6 y^{2}$; (middle) surface rings 3,4,5 of [Loo02]; (right) surface rings 3,4,5 of $\mathbf{x}_{L 4}$. (bottom row) Gaussian curvature shading: the $C^{2}$ construction $\mathbf{x}_{L 8}$ (for comparison), [Loo02], $\mathbf{x}_{L 4}$. 